Article

\title{
Organic Dye-Doped PMMA Lasing
}

\author{
Pen Yiao Ang ${ }^{1}$, Marko Čehovski ${ }^{1}$, Frederike Lompa ${ }^{1}$, Christian Hänisch ${ }^{2} \mathbb{D}$, Dinara Samigullina ${ }^{2} \mathbb{D}$, \\ Sebastian Reineke ${ }^{2}$, Wolfgang Kowalsky ${ }^{1,3}$ and Hans-Hermann Johannes ${ }^{1,3, *(D)}$
}

check for updates

Citation: Ang, P.Y.; Čehovski, M.; Lompa, F.; Hänisch, C.; Samigullina, D.; Reineke, S.; Kowalsky, W.; Johannes, H.-H. Organic Dye-Doped PMMA Lasing. Polymers 2021, 13, 3566. https://doi.org/10.3390/ polym13203566

Academic Editor: Esther Rebollar

Received: 30 August 2021

Accepted: 8 October 2021

Published: 15 October 2021

Publisher's Note: MDPI stays neutral with regard to jurisdictional claims in published maps and institutional affiliations.

Copyright: (c) 2021 by the authors. Licensee MDPI, Basel, Switzerland. This article is an open access article distributed under the terms and conditions of the Creative Commons Attribution (CC BY) license (https:// creativecommons.org/licenses/by/ $4.0 /)$.
1 Institut für Hochfreuquenztechnik, Technische Universität Braunschweig, Schleinitzstraße 22, 38106 Braunschweig, Germany; pen.yiao.ang@ihf.tu-bs.de (P.Y.A.); marko.cehovski@ihf.tu-bs.de (M.Č.); f.lompa@tu-braunschweig.de (F.L.); wolfgang.kowalsky@ihf.tu-bs.de (W.K.)

2 Dresden Intergrated Center for Applied Physics and Photonic Materials, Technische Universität Dresden, Nöthnitzer Straße 61, 01187 Dresden, Germany; christian.haenisch@tu-dresden.de (C.H.); dinara.samigullina@tu-dresden.de (D.S.); sebastian.reineke@tu-dresden.de (S.R.)

3 Cluster of Excellence PhoenixD (Photonics, Optics, and Engineering-Innovation across Disciplines), 30167 Hannover, Germany

* Correspondence: h2.johannes@ihf.tu-bs.de

\begin{abstract}
Organic thin-film lasers gain interest as potential light sources for application in diverse fields. With the current development, they hold variety of benefits such as: low-cost, highperformance, and color-tunability. Meanwhile, the production is not complicated because both the resonator and the gain medium can be assembled by solution-processable organic materials. To our knowledge, information about using poly(methyl methacrylate) (PMMA) as a matrix for organic dye lasers was insubstantial. Herein, the feasibility of using organic dye-doped PMMA as an organic dye laser was tested. Six different sample designs were introduced to find out the best sample model. The most optimum result was displayed by the sample design, in which the gain medium was sandwiched between the substrate and the photoresist layer with grating structure. The impact of dye concentration and grating period on peak wavelength was also investigated, which resulted in a shift of $6 \mathrm{~nm}$ and $25 \mathrm{~nm}$, respectively. Moreover, there were in total six various organic dyes that could function well with PMMA to collectively perform as 'organic dye lasers', and they emitted in the range of $572 \mathrm{~nm}$ to $609 \mathrm{~nm}$. Besides, one of the samples was used as a sensor platform. For instance, it was used to detect the concentration of sugar solutions.
\end{abstract}

Keywords: organic laser; polymer laser; laser tuning

\section{Introduction}

Organic dye laser is a challenging research topic because it provides a new horizon for simple, low-cost, time-saving, versatile, and environmental-friendly fabrication of new and desirable laser structures. Diverse application fields have been found, such as lab-on-chip spectroscopy [1], data/optical communication [2], refractive index sensor [3], vapour pressure detector [4], absorption and transmission spectroscopy [5,6]. Distributedfeedback (DFB) lasers are fabricated by introducing grating structure to organic dye-doped lasers, and this type of lasers can provide single-mode emission with narrow bandwidth. Generally, two different methods are followed to design a DFB polymer thin-film laser. Firstly, a grating structure can directly be written into the polymer/gain medium using electron-beam lithography or holographic lithography [7-12]. Secondly, the gain material can either be solution-processed (e.g., spin-coating and dip-coating) or vacuum deposited on the grating structure [13-18]. The lasing wavelength of a DFB thin-film laser can be easily tuned by applying electrical control [19], modulating the thickness of the active layer [20,21], stretching the active film [22-24], designing a wedge-shaped film [12,25], applying different grating periods [26,27] and using a liquid crystal [28,29]. 
To function as an organic gain media, organic dyes have been dissolved in liquid solvents for the lasing purpose for decades [30]. However, the organic liquid laser can induce unnecessary evaporation because of their solvent. To solve this problem, organic solid-state lasers can be generated. Generally, the gain medium is categorised into two main groups. First group includes the polymer which is a fluorophore and it can directly be used as a gain medium [31-33]. Second group of gain medium requires doping of several organic dyes into non-conjugated polymers. By using this method, the devices can be fabricated to be mechanically flexible and the preparation can be non-expensive by using solution-based methods. The typically applied polymers that are mentioned in the literature are polystyrene (PS) and PMMA. Most of these studies proposed different DFB thin-film architectures by using PS as a matrix [34,35]. On the other hand, PMMA was mostly found to be optical fiber [36-38] or fiber laser [39,40]. Thin-film based on dyedoped PMMA [41,42] showed a Full-Width-at-Half-Maximum (FWHM) as big as $10 \mathrm{~nm}$. Literature regarding the integration of grating structure on organic dye-doped PMMA film can be barely found.

In this study, we demonstrate the feasibility of organic dye-doped PMMA thin-film laser by analysing different sample designs with PMMA as a matrix by using the solutionprocessed method, spin-coating. In the results section, constriction of FWHM of the spectra and clear lasing threshold are shown. Modifications of the gain medium and the resonator were carried out to demonstrate the lasing state. Furthermore, the workability of a set of 6 different alternative organic dyes was evaluated. Moreover, the application potential as a refractive index sensor was presented as well.

\section{Results}

\subsection{Design Variation}

To find out the sample model with the best lasing performance, six different sample designs were introduced. Rhodamine 6G (Rh6G) was selected as the first dye dopant in this experiment because it is commonly found in the literature for PMMA fiber laser [43-45]. The concentration of Rh6G in PMMA was fixed at $400 \mathrm{ppm}$. In the first design (D1), a flat and single-layer of dye-doped PMMA was spin-coated on the Si-Substrate. Next, a grating structure was proposed directly on the layer of dye-doped PMMA in the second design (D2). In the third design (D3), a layer of photoresist, EpoClad was generated on top of the dye-doped PMMA thin-film. Furthermore, an EpoClad layer was also spin-coated on top of the dye-doped PMMA with a grating structure in the fourth design (D4). In the fifth design (D5), a flat dye-doped PMMA layer was sandwiched between the substrate and the grating structured EpoClad layer. In the sixth design (D6), a layer of EpoClad with a grating structure was produced on top of the substrate and covered by a spin-coated dye-doped PMMA layer. D6 is commonly shown in various publications $[7,13,15,21,32]$.

When the grating structure was introduced to the sample design, the required grating periods were calculated by using the following equation:

$$
\lambda_{\text {Bragg }}=\left(\frac{2 n_{e f f} \Lambda}{m}\right)
$$

where $n_{e f f}$ is the effective refractive index of dye-doped PMMA, $m$ is the order of diffraction and $\Lambda$ is the grating period. $\lambda_{\text {Bragg }}$ is the resonant wavelength in the cavity, which is reinforced during its propagation along the active layer before it is diffracted in the grating across different directions. Second order of diffraction $(m=2)$ was applied here.

Other than this, the grating period of both PMMA layer and EpoClad layer was intended to be $380 \mathrm{~nm}$ as per the peak wavelength of the emission spectrum of Rh6G. Meanwhile, the measured average grating period on top of the PMMA layer was $379.85 \mathrm{~nm}$, while it was $380.16 \mathrm{~nm}$ on top of the EpoClad layer (see Figure 1). The result was significantly close to the desired value. Therefore, it can be deduced that the grating structure with the expected grating period can be properly built on top of the PMMA layer and the EpoClad layer. 
(a)

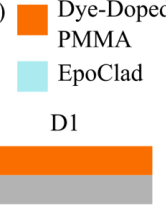

D3

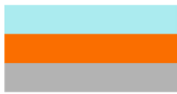

D5
Si Substrate

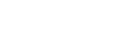

$\mathrm{D} 2$

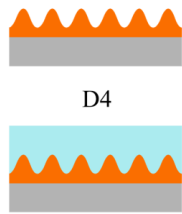

D6 (b)

PMMA

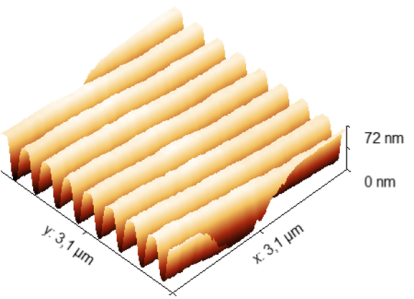

(c)

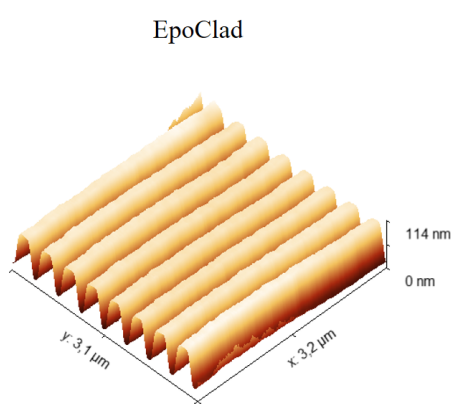

Figure 1. (a) Six different sample designs were produced to find out the best sample model. The grating profile on the (b) PMMA layer and the (c) EpoClad layer were observed.

The result of lasing properties due to design variation is shown in Figure 2. The photoluminescence spectrum of the Rh6G doped sample has a FWHM of about $36 \mathrm{~nm}$. By using D1, the FWHM was reduced to $9.01 \mathrm{~nm}$. According to the study of Samuel, I.D. et al. [46], the sample achieved the state of amplified stimulated emission (ASE) because values of FWHM of ASE are typically around $10 \mathrm{~nm}$. Therefore, it could be said that D1 reached ASE but not lasing state. With the application of the grating structure, the FWHM of D2 was only $1.16 \mathrm{~nm}$. Meanwhile, by using D3, the FWHM was $2.90 \mathrm{~nm}$. This meant that the grating structure had a strong effect on the constriction of FWHM compared to the EpoClad layer. However, when the EpoClad layer was generated on top of the grating structure, the FWHM of D4 became $2.18 \mathrm{~nm}$. This indicates that EpoClad could not improve the FWHM when the grating structure was directly applied on the gain medium. Among the samples, D5 had the smallest FWHM $(0.83 \mathrm{~nm})$. This design was suggested by Zhai, T. et al. [31], who proposed the idea that direct structuring on the gain medium would cause uneven distribution of light modes along the gain medium. With D5, the light mode was distributed completely and it helped in improving the lasing properties. By using D6, the FWHM became slightly larger $(1.97 \mathrm{~nm})$, which could be caused by the direct interaction of the gain medium and the excitation source. In such a way, the light could not be confined well in the gain medium.

For the aspect of peak wavelength, D1 and D3 were found to have nearly same value. This is because the designs of D1 and D3 were nearly the same and both had the same layer thickness of Rh6G doped PMMA. On the other hand, D2 and D4 had the same peak wavelength because the layer thickness of dye-doped PMMA of both designs was the same. D5 and D6 had a thicker dye-doped PMMA layer, therefore the peak wavelength was detected at a longer wavelength. Hence, it can be deduced that the peak wavelength was red-shifted to a longer wavelength with an increasing layer thickness of dye-doped PMMA. There are more dye dopants with thicker layer thickness. There is a high possibility of reabsorption of the emission of the dopants. The red-shift might be caused by the re-emission of the reabsorbed photons.

The lasing threshold of the samples was investigated by exciting them with a series of pump energies. The turning point, in which the light was amplified, was noticed as the lasing threshold. By using D1, the lasing threshold was the lowest among the designs $0.131 \mu \mathrm{J}$. This was due to the fact that there was no additional optical system like a grating structure or an EpoClad layer. With the application of a grating structure or an EpoClad layer like in D2 or D3, the lasing threshold increased on a small scale (approx. 0.2-0.3 $\mathrm{J}$ ). When the combination of the grating and EpoClad layer was applied like in D4, D5, and D6, the lasing threshold raised further $(>0.3 \mu \mathrm{J})$. It can be deduced here that the lasing threshold increased when the grating and/or EpoClad layer was implemented. 
(a)

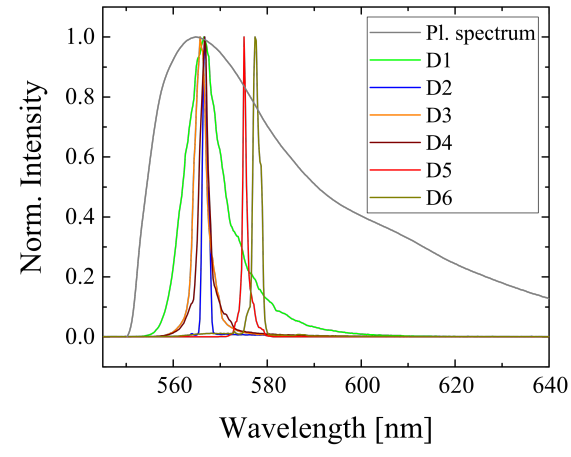

(1)

\begin{tabular}{|c|r|r|r|r|r|r|r|}
\hline Design & $\begin{array}{c}\text { Thickness } \\
\text { of PMMA } \\
\text { /nm }\end{array}$ & $\begin{array}{c}\text { Thickness } \\
\text { of EpoClad } \\
/ \mathrm{nm}\end{array}$ & $\begin{array}{c}\text { Peak } \\
\text { wavelength } \\
/ \mathrm{nm}\end{array}$ & $\begin{array}{c}\text { FWHM } \\
/ \mathrm{nm}\end{array}$ & $\begin{array}{c}\text { Lasing } \\
\text { threshold } \\
/ \mu \mathrm{J}\end{array}$ & $\begin{array}{c}\text { PER } \\
/ \mathrm{dB}\end{array}$ & $\begin{array}{c}\text { Output } \\
\text { energy } \\
/ \mathrm{nJ}\end{array}$ \\
\hline D1 & 1200 & - & 567 & 9.01 & 0.131 & 21.30 & 3.63 \\
D2 & 702 & - & 567 & 1.16 & 0.271 & 24.57 & 1.38 \\
D3 & 1200 & 782 & 566 & 2.90 & 0.206 & 24.53 & 8.56 \\
D4 & 702 & 857 & 567 & 2.18 & 0.356 & 28.12 & 6.00 \\
D5 & 1200 & 661 & 575 & 0.83 & 0.311 & 30.88 & 16.43 \\
D6 & 1280 & 765 & 577 & 1.97 & 0.353 & 23.49 & 8.82 \\
\hline
\end{tabular}

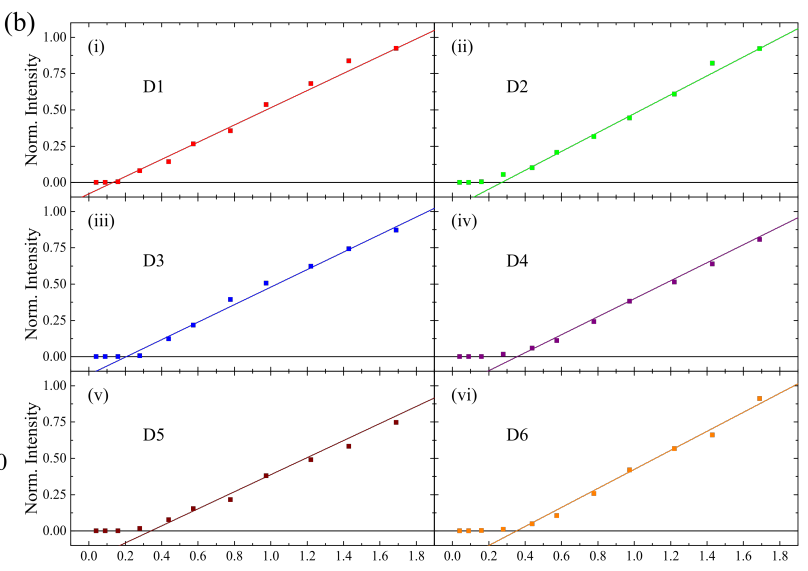

Pump Energy $[\mu \mathrm{J}]$

Figure 2. (a) The photoluminescence spectrum of Rh6G doped thin-film and the lasing spectra of design variation were shown. (b) The lasing threshold of different sample designs were evaluated. An overview of the lasing properties under the influence of the changes of sample design was presented in (c).

By comparing the polarisation extinction ratio (PER) between the samples, the value of D1 was the smallest $(21.30 \mathrm{~dB})$. The result of D2 $(24.57 \mathrm{~dB})$ and D3 $(24.53 \mathrm{~dB})$ showed that the grating structure and the EpoClad layer helped in improving the PER with the same strength. With the combination of the grating structure and the EpoClad layer, the PER can be further improved like in D4 (28.12 dB) and D5 (30.88 dB). D5 had the most optimum PER because the distribution of light mode along the gain medium was thorough without disturbance of the grating structure. By using D6, the PER $(23.49 \mathrm{~dB})$ did not show the highest value because the gain medium was in direct contact with the excitation source.

Among the samples, D2 had the lowest output energy $(1.38 \mathrm{~nJ})$, which was due to the fact that the layer thickness of PMMA was the narrowest. With a flat thin-film, D1 had an output energy of 3.63 nJ, which is higher than that of D2's because the PMMA layer of D1 was thicker. Therefore, the thicker the gain medium, the higher the output energy. By adding an EpoClad layer on it like in D4, the output energy was improved to $6.00 \mathrm{~nJ}$. With an additional EpoClad layer on the pure thin-film like in D3, the output energy was enhanced to 8.56 nJ. Here, it can be deduced that an additional EpoClad layer can help in improving the output energy, by providing extra reflectivity due to the difference of refractive index. D5 had the highest output energy (16.43 nJ) because the light was amplified well through the grating structure and the EpoClad layer. On the other hand, by using D6, the output energy was $8.82 \mathrm{~nJ}$ because there was no extra optical structure on top of the gain medium for the light amplification.

Even though the lasing threshold of D5 is not the smallest, it was chosen as the best sample model because it provided the best lasing performance. D5 had the smallest FWHM (0.83 nm), the highest PER (30.88 dB), and the highest output energy (16.43 nJ). The peak wavelength of D5 was detected at $575 \mathrm{~nm}$. For these aforementioned reasons, D5 was selected for further experiments. 


\subsection{Concentration Variation}

Any changes in the gain medium will affect the lasing properties. To assure the laser state, the property of the gain medium was manipulated. Here, three different concentrations of Rh6G were examined, which were 100 ppm, 200 ppm, and 400 ppm, while the grating period was set at $380 \mathrm{~nm}$. The result is displayed in Figure 3. The FWHM of 100 ppm Rh6G doped sample was $10.85 \mathrm{~nm}$, which indicated that only ASE were attained and no laser state was reached because $100 \mathrm{ppm}$ concentration was too low, thus fewer dye molecules could contribute to laser generation. When the concentration of Rh6G was increased to $200 \mathrm{ppm}$ and $400 \mathrm{ppm}$, the FWHM was $<1 \mathrm{~nm}$, which were $0.89 \mathrm{~nm}$ and $0.83 \mathrm{~nm}$, respectively. At the same time, the peak wavelengths of $200 \mathrm{ppm}$ and $400 \mathrm{ppm}$ were $569 \mathrm{~nm}$ and $575 \mathrm{~nm}$, subsequently. There was a $6 \mathrm{~nm}$ red-shift of peak wavelength when the Rh6G concentration was increased from $200 \mathrm{ppm}$ to $400 \mathrm{ppm}$. It could be explained that by the presence of more dyes in a higher concentration, which had a higher reabsorption possibility and induced the red-shift eventually.
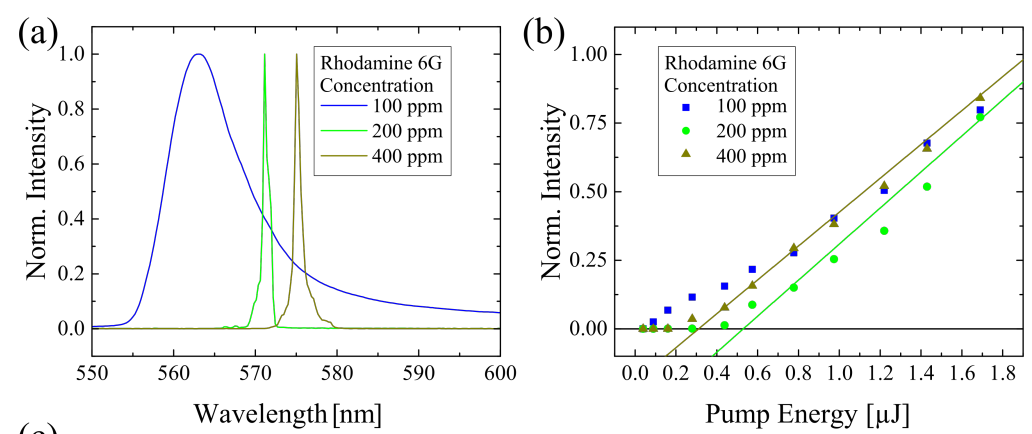

(c)

\begin{tabular}{|r|r|r|r|r|r|}
\hline $\begin{array}{c}\text { Rh6G } \\
\begin{array}{c}\text { Concentration } \\
/ \mathrm{ppm}\end{array}\end{array}$ & $\begin{array}{c}\text { Peak } \\
\text { wavelength } \\
/ \mathrm{nm}\end{array}$ & $\begin{array}{c}\text { FWHM } \\
/ \mathrm{nm}\end{array}$ & $\begin{array}{c}\text { Lasing } \\
\text { threshold } \\
/ \mu \mathrm{J}\end{array}$ & $\begin{array}{c}\text { PER } \\
/ \mathrm{dB}\end{array}$ & $\begin{array}{c}\text { Output } \\
\text { energy } \\
/ \mathrm{nJ}\end{array}$ \\
\hline 100 & 563 & 10.85 & & 15.58 & 0.22 \\
200 & 569 & 0.89 & 0.530 & 28.94 & 6.12 \\
400 & 575 & 0.83 & 0.311 & 30.88 & 16.43 \\
\hline
\end{tabular}

Figure 3. By using different Rhodamine 6G concentrations (100 ppm, 200 ppm, and 400 ppm), (a) the lasing spectra and (b) the lasing threshold were determined. (c) A table was presented to provide an overview of the lasing properties under the influence of Rhodamine $6 \mathrm{G}$ concentration variation.

By using a 100 ppm Rh6G doped sample, no lasing threshold can be seen. This confirms again that the sample did not achieve the lasing state. The lasing threshold of the $200 \mathrm{ppm}$ Rh6G doped sample $(0.530 \mu \mathrm{J})$ was higher than the $400 \mathrm{ppm}$ Rh6G doped sample. This is due to the fact that there was less dye in $200 \mathrm{ppm}$ compared to $400 \mathrm{ppm}$. Therefore, more energy was needed to obtain lasing state. The same explanation can be used to understand the trend of the output energy. The higher the concentration is, the higher would be the output energy. When the concentration was higher, there were more dye molecules in the sample, which could contribute to the output energy. For the aspect of PER, the value was about $>28 \mathrm{~dB}$, once the sample attains the lasing state.

\subsection{Grating Variation}

On top of the changes of the gain medium, any changes of the resonator will alter the lasing properties. Another method to confirm the lasing state was to modify the resonator. In this case, the grating structure was the resonator of D5. Three different grating periods $(370 \mathrm{~nm}, 380 \mathrm{~nm}$, and $390 \mathrm{~nm}$ ) were applied to check its influence on the lasing properties, while the Rh6G concentration was fixed at 400 ppm. The result is illustrated in Figure 4. A $25 \mathrm{~nm}$ wavelength-shift has been observed by using the three aforementioned grating periods. The measured peak wavelengths were $564 \mathrm{~nm}, 575 \mathrm{~nm}$, and $589 \mathrm{~nm}$, respectively. The FWHM of all the spectra was found to be $<1 \mathrm{~nm}$. On top of this, it has also been observed that the lasing threshold decreased when a smaller grating period was applied. 
The reason could be that the optical gain increased with a smaller grating period (see Figure 4a). Meanwhile, the higher the optical gain, the higher the stimulated emission cross section [47]. Miniscalco, W. et al. suggested that the lasing threshold became smaller when the stimulated emission cross section was higher [48]. On the other hand, the output energy became higher with increasing stimulated emission cross section, when the applied grating period was smaller. Besides, it is observed that the PER was quite high $(>28 \mathrm{~dB})$, which was not strongly affected by the changes of the grating period.
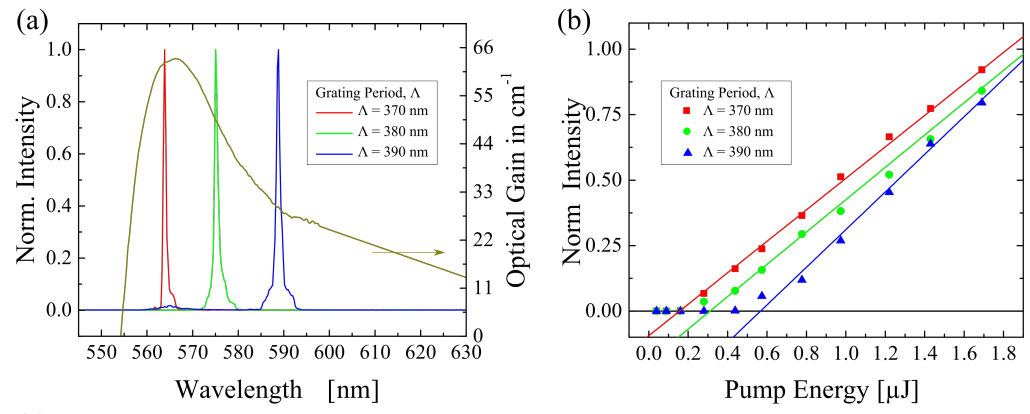

\begin{tabular}{|c|c|c|c|c|c|}
\hline $\begin{array}{l}\text { Grating } \\
\text { period } \\
\text { /nm }\end{array}$ & $\begin{array}{c}\text { Peak } \\
\text { wavelength } \\
/ \mathrm{nm}\end{array}$ & $\begin{array}{l}\text { FWHM } \\
\text { /nm }\end{array}$ & $\begin{array}{c}\text { Lasing } \\
\text { threshold } \\
/ \mu \mathrm{J}\end{array}$ & $\begin{array}{c}\text { PER } \\
/ \mathrm{dB}\end{array}$ & $\begin{array}{c}\text { Output } \\
\text { energy } \\
\text { /nJ }\end{array}$ \\
\hline 370 & 564 & 0.70 & 0.156 & 30.97 & 17.13 \\
\hline 380 & 575 & 0.83 & 0.311 & 30.88 & 16.43 \\
\hline 390 & 589 & 0.95 & 0.566 & 28.27 & 11.78 \\
\hline
\end{tabular}

Figure 4. When the grating period ( $370 \mathrm{~nm}, 380 \mathrm{~nm}$, and $390 \mathrm{~nm}$ ) was altered, (a) the lasing spectra and (b) the lasing threshold were defined. While, the summary of the result of lasing properties was presented in (c).

\subsection{Dyes Variation}

The result shown so far was obtained by using the Rh6G doped sample. It is important to check the workability of other organic dyes together with PMMA. It has been observed that six different organic dyes showed positive results, which were Rh6G, Rhodamine B (RhB), Lumogen Orange (LumO), Pyrromethene 597 (P597), 4-(Dicyanomethylene)-2-tertbutyl-6-(1,1,7,7-tetramethyljulolidin-4-yl-vinyl)-4H-pyran (DCJTB) and 4-(Dicyanomethylene)2-methyl-6-julolidyl-9-enyl-4H-pyran (DCM2). The concentration of these dyes was set at $400 \mathrm{ppm}$, while the applied grating period was calculated according to Equation (1) using the peak wavelength of the respective photoluminescence spectra (see Appendix A). The result is elaborated in Figure 5. The lasing spectra of these dye-doped samples were found to be in the range from $572 \mathrm{~nm}$ to $609 \mathrm{~nm}$. All the FWHMs were found to be $<1 \mathrm{~nm}$. Meanwhile, all the samples had relatively high PER ( $>28 \mathrm{~dB})$. Among the samples, P597 has a peak wavelength of $572 \mathrm{~nm}$ with the smallest FWHM $(0.57 \mathrm{~nm})$, the lowest lasing threshold $(0.253 \mu \mathrm{J})$, the highest output energy (18.04 nJ), and the longest sample lifetimes (33,000 pump pulses).

\subsection{Application Potential}

Since the P597 doped sample showed the best performance, the sample model was modified by attaching a cover to it for application possibility (see Figure 6). In this way, solutions with different sugar concentrations were injected into it for checking their influence on the lasing spectra. With increasing sugar concentration, the peak wavelength was red-shifted to a longer wavelength. When the sugar concentration was more than $20 \mathrm{wt} \%$, smaller peaks with lower intensity were detected. This could be due to the injected sugar which tended to re-crystallise because of the increasing sugar concentration. A high sugar concentration corresponds to a high refractive index. It was observed that, when the sugar concentration increased, the refractive index went up (see Appendix A), and the peak wavelength of the lasing spectra increased exponentially. This indicated that the sample can be used as a refractive index sensor caused the sugar concentration. 
(a)

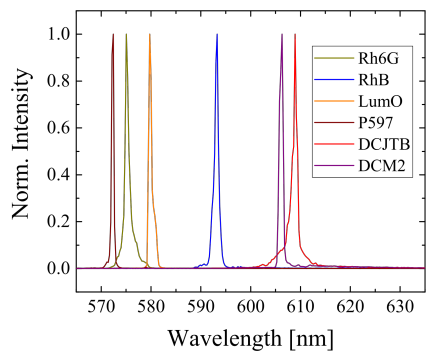

(c)

\begin{tabular}{|c|r|r|r|r|r|r|r|}
\hline Dye & $\begin{array}{c}\text { Grating } \\
\text { period } \\
/ \mathrm{nm}\end{array}$ & $\begin{array}{c}\text { Peak } \\
\text { wavelength } \\
/ \mathrm{nm}\end{array}$ & $\begin{array}{c}\text { FWHM } \\
/ \mathrm{nm}\end{array}$ & $\begin{array}{c}\text { Lasing } \\
\text { threshold } \\
/ \mu \mathrm{J}\end{array}$ & $\begin{array}{c}\text { PER } \\
/ \mathrm{dB}\end{array}$ & $\begin{array}{c}\text { Output } \\
\text { energy } \\
/ \mathrm{nJ}\end{array}$ & $\begin{array}{c}\text { Sample } \\
\text { lifetimes } \\
/ \text { pump } \\
\text { pulses }\end{array}$ \\
\hline $\mathrm{Rh} 6 \mathrm{G}$ & 380 & 575 & 0.83 & 0.311 & 30.88 & 16.43 & 16000 \\
$\mathrm{RhB}$ & 390 & 593 & 0.81 & 0.775 & 30.65 & 12.42 & 20000 \\
LumO & 390 & 580 & 0.70 & 0.454 & 30.87 & 14.01 & 23000 \\
P597 & 380 & 572 & 0.57 & 0.253 & 31.12 & 18.04 & 33000 \\
DCJTB & 400 & 609 & 0.90 & 1.254 & 28.06 & 5.80 & 2500 \\
DCM2 & 400 & 606 & 0.93 & 1.227 & 28.01 & 6.18 & 3000 \\
\hline
\end{tabular}

(b)

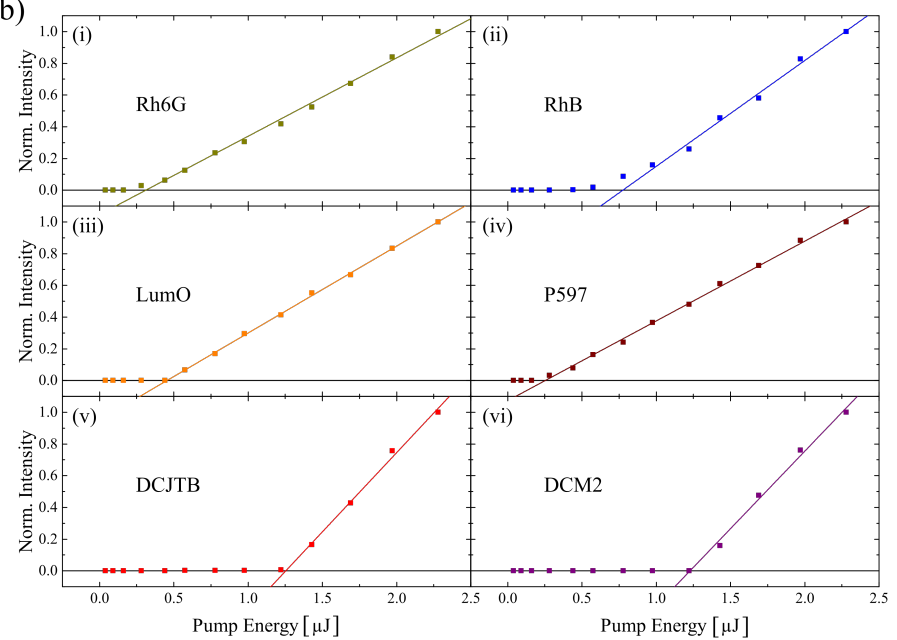

Figure 5. Six different organic dyes were found to be good dopants for PMMA. (a) The lasing spectra and (b) the lasing threshold of the respective dye-doped PMMA were measured. Meanwhile, an overview of the result was demonstrated in (c).

(a)

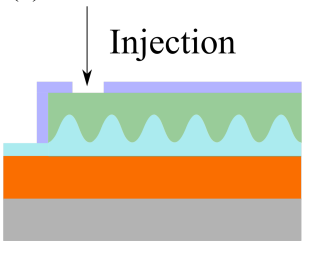

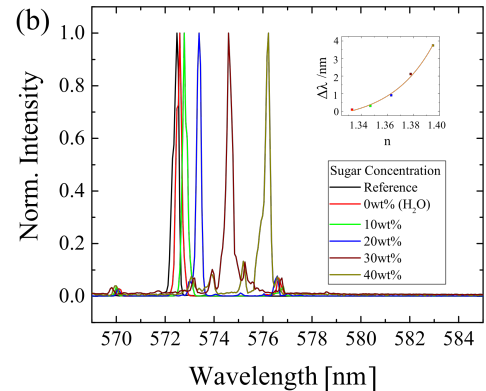

Figure 6. (a) The sample model was modified to detect the solution with different sugar concentration. (b) The lasing spectra under the influence of different sugar concentration were determined.

\section{Conclusions}

In this work, the feasibility of using PMMA as a matrix with a grating structure to function as a thin-film laser was proven. At first, six different sample designs were introduced to find out the best sample model as dye-doped PMMA thin-film laser. The design, in which the gain medium was sandwiched between the substrate and the EpoClad layer with grating structure, provided the best performance. Moreover, the lasing state of the sample was re-established by alternating the gain medium and the resonator, in which the dye concentration and the grating period were varied. By using the Rh6G concentrations from $100 \mathrm{ppm}$ to $400 \mathrm{ppm}$, a peak wavelength shift of $6 \mathrm{~nm}$ is observed. Meanwhile, by modifying the grating periods from $370 \mathrm{~nm}$ to $390 \mathrm{~nm}$, a peak wavelengthshift of $25 \mathrm{~nm}$ was also observed. Besides, a total six various dyes were confirmed to function well together with PMMA as organic dye lasers, namely, Rh6G, RhB, LumO, P57, DCJTB, and DCM2. The peak wavelengths of these different dye-doped samples were in the range of $572 \mathrm{~nm}$ to $609 \mathrm{~nm}$. With the best dye option, the P597 dye-doped 
PMMA sample was modified to examine solutions with different sugar concentrations, by observing the shift of peak wavelength according to the changes of the refractive index of the sugar concentrations. Therefore, it can be concluded that the workability of dye-doped PMMA as a thin-film laser with application potential is confirmed.

\section{Methods and Materials}

PMMA used in this research, PLEXIGLAS ${ }^{\circledR} 8 \mathrm{~N}$, was purchased from the company, Röhm GmbH, Darmstadt, Germany. It was supplied in uniform size as pellets. To dope different dyes in PMMA, PMMA pellets together with the dyes were dissolved in butanone (purchased from Sigma-Aldrich Chemie $\mathrm{GmbH}$, Taufkirchen, Germany). The concentration of the PMMA concerning to butanone was kept at $7.5 \mathrm{wt} \%$, while the concentration of the dyes was determined with respect to PMMA. Rh6G, RhB, P597, and DCJTB were purchased from Radiant, Wermelskirchen, Germany, while LumO and DCM2 were purchased from TCI, Tokyo, Japan. EpoClad (purchased from micro resist technology $\mathrm{GmbH}$, Berlin, Germany) was diluted with gamma-Butyrolactone into $50 \mathrm{wt} \%$ before being used.

Since both PMMA and EpoClad were solution-processable materials, the technique of spin-coating was applied. To generate a grating structure directly on the PMMA surface, a stamp with the appropriate grating structure was placed on top of the PMMA thinfilm. Together with the stamp, the sample was heated at $130^{\circ} \mathrm{C}$ under a pressure of $6.125 \mathrm{mN} \mathrm{mm}^{-2}$ for $25 \mathrm{~min}$.

To cure EpoClad, an EpoClad layer was spin-coated in advance. Then, the EpoClad layer was pre-baked at $120^{\circ} \mathrm{C}$ for $5 \mathrm{~min}$. Afterwards, it was cured under UV light for $20 \mathrm{~s}$. Then, it was post-baked at $120^{\circ} \mathrm{C}$ for $3 \mathrm{~min}$. The progress was preformed together with a stamp if the grating structure was established on top of the EpoClad layer.

To measure the optical gain, the variable stripe length method was applied. The setup is demonstrated in Figure 7a. A Nd:YAG laser (STA-01-1-1SH purchased from Stand, Vilnius, Lithuania) was implemented. The pump intensity was controlled by a Glan-Thomson polariser. Before reaching the sample, the pump intensity passed through a few lenses and aperture to assure a homogenous excitation line (see Figure $7 \mathrm{~b}$ ). The sample surface from an edge was excited perpendicularly to avoid the losses due to total internal reflection. For the optical gain measurement, the length of excitation was varied to avoid light saturation.
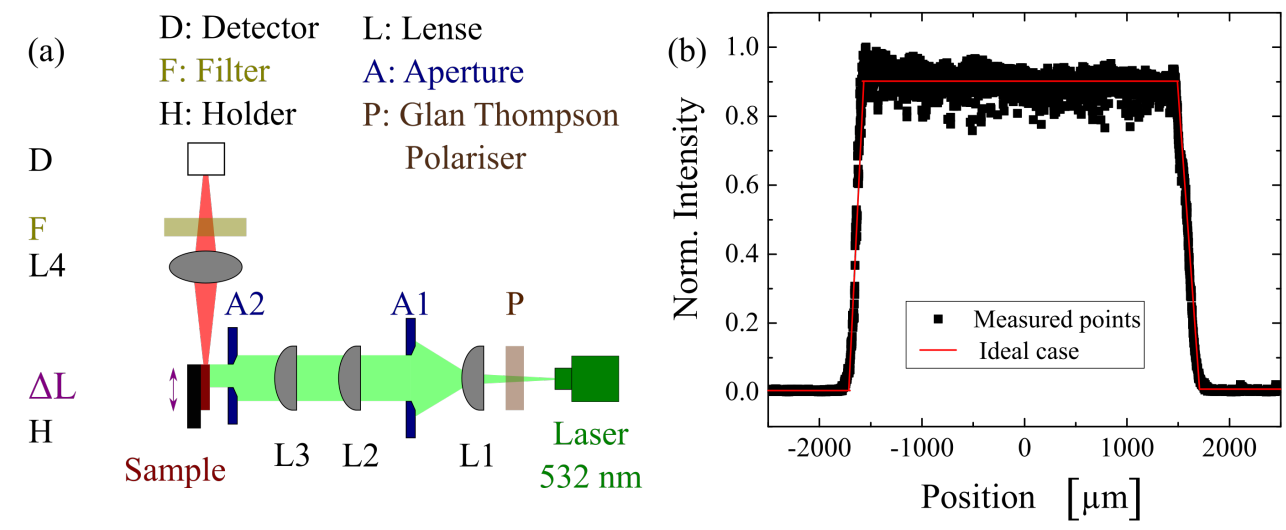

Figure 7. (a) The setup of the optical gain measurement and the measurement of lasing properties, and (b) the intensity profile of the pump light were shown. 
For the measurement of lasing properties, the setup was identical to the setup of the variable stripe length method. The length of excitation was fixed at $2.3 \mathrm{~mm}$. The emission from the sample was collected for evaluation. The detector used was either a spectrometer (Triax320 purchased from Jobin Yvon, Bensheim, Germany) or a powermeter (LabMax ${ }^{\mathrm{TM}}$-TOP purchased from Coherent, Santa Clara, CA, USA). For the measurement of PER, the rotating polariser method was used. A polariser (LPVISE100-A purchased from Thorlabs, Bergkirchen, Germany) was established in front of the detector. Spectra were taken at $0^{\circ}$ and $90^{\circ}$. The maximum values of each spectrum were compared using the following equation:

$$
P E R=10 \log _{10} \frac{P_{\max }}{P_{\min }} .
$$

Author Contributions: P.Y.A. had the idea, performed the experiments, evaluated the data and created all figures. M.Č. helped with the experiments, discussed the results and built the setup for optical gain. F.L. produced the samples. D.S. measured the roughness and the grating profile of the PMMA layer and the EpoClad layer. C.H. measured the refractive index of the PMMA layer and the EpoClad layer. S.R. discussed and verified the result from D.S. and C.H., H.-H.J. discussed and verified the result and organised the collaboration. W.K. oversaw the complete project. P.Y.A. wrote the manuscript under the supervision of H.-H.J. All authors have read and agreed to the published version of the manuscript.

Funding: The work was supported by grants from Niedersächsische Ministerium für Wissenschaft und Kultur in LaPOF research network (EFRE-SER 85003655) and from two projects of Deutsche Forschungsgemeinschaft: DFG Cluster of Excellence PhoenixD (Exc 2122, Project ID 390833453) and DFG-project PolySens (AOBJ: 654666).

Institutional Review Board Statement: Not applicable.

Informed Consent Statement: Not applicable.

Data Availability Statement: The data in this work are available upon request from the corresponding author.

Conflicts of Interest: The authors declare no conflict of interest.

\section{Appendix A}

The roughness of the PMMA layer and the EpoClad layer was determined to be $<1 \mathrm{~nm}$, which was considered to be very smooth and suitable for lasing research. Besides, the measured refractive indices of PMMA and EpoClad were 1.49 and 1.58, which agreed with the data from the supplier.

The absorption spectra and the emission spectra of the used dyes were presented in Figure A1. The peak wavelength of the respective emission spectra was used to calculated the applied grating periods using Equation (1) with the consideration that the refractive index of PMMA was 1.49.

According to Malinin, A. et al. [49], the theoretical value of the refractive index of the sugar concentration $\left(n_{\text {sugar }}\right)$ can be calculated using the equation below:

$$
n_{\text {sugar }}=n_{w}-a C .
$$

where, $n_{w}$ is the refractive index of water at $25^{\circ} \mathrm{C}\left(n_{w}=1.3324\right.$ at $\left.589 \mathrm{~nm}\right), a$ denotes a specific increment of a refraction (0.00143) and $C$ is sugar concentration in $\mathrm{g} / 100 \mathrm{~mL}$ or $w t \%$. The experimental value of the refractive index of sugar solution was accomplished by using the refractometric method. An Atago refractometer with $589 \mathrm{~nm}$ as incident light was applied. 

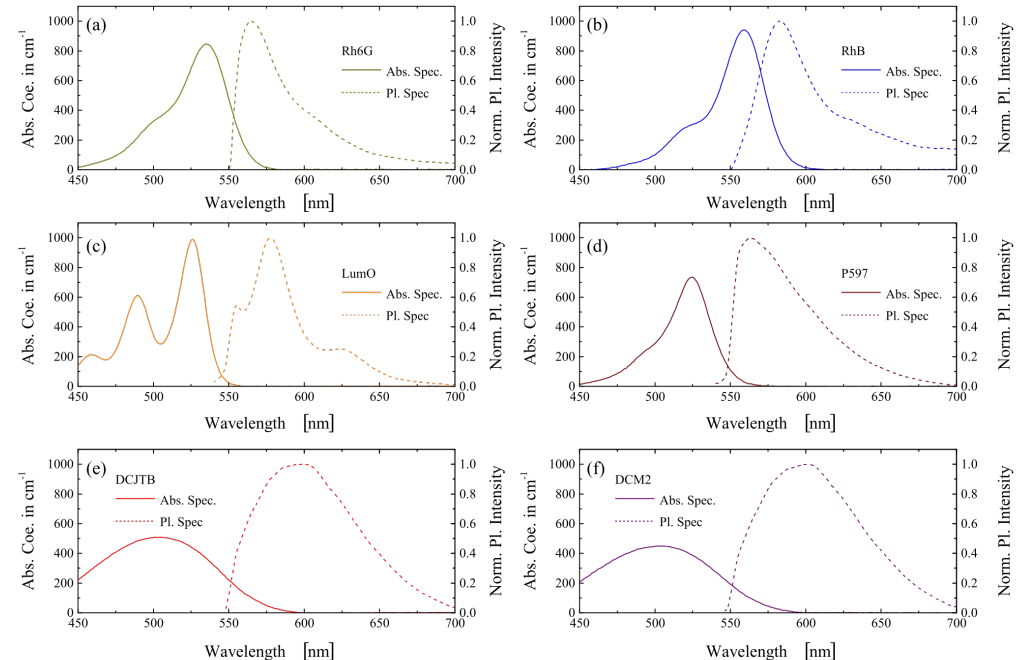

Figure A1. The absorption spectra and the emission spectra of dye-doped PMMA were illustrated: (a) Rh6G, (b) RhB, (c) LumO, (d) P597, (e) DCJTB and (f) DCM2.

\section{References}

1. Vannahme, C.; Klinkhammer, S.; Lemmer, U.; Mappes, T. Plastic lab-on-a-chip for fluorescence excitation with integrated organic semiconductor lasers. Opt. Express 2011, 19, 8179-8186.

2. Clark, J.; Lanzani, G. Organic photonics for communications. Nat. Photon. 2010, 4, 438-446.

3. Morales-Vidal, M.; Boj, P.G.; Quintana, J.A.; Villalvilla, J.M.; Retolaza, A.; Merino, S.; Díaz-García, M.A. Distributed feedback lasers based on perylenediimide dyes for label-free refractive index sensing. Sens. Actuators B Chem. 2015, 220, 1368-1375.

4. Wang, Y.; Morawska, P.O.; Kanibolotsky, A.L.; Skabara, P.J.; Turnbull, G.A.; Samuel, I.D. LED pumped polymer laser sensor for explosives. Laser Photon. Rev. 2013, 7, L71-L76.

5. Oki, Y.; Miyamoto, S.; Maeda, M.; Vasa, N.J. Multiwavelength distributed-feedback dye laser array and its application to spectroscopy. Opt. Lett. 2002, 27, 1220-1222.

6. Woggon, T.; Klinkhammer, S.; Lemmer, U. Compact spectroscopy system based on tunable organic semiconductor lasers. Appl. Phys. B 2010, 99, 47-51.

7. Ramírez, M.G.; Villalvilla, J.M.; Quintana, J.A.; Boj, P.G.; Díaz-García, M.A. Distributed feedback lasers based on dichromated poly (vinyl alcohol) reusable surface-relief gratings. Opt. Mater. Express 2014, 4, 733-738.

8. Nilsson, D.; Nielsen, T.; Kristensen, A. Solid state microcavity dye lasers fabricated by nanoimprint lithography. Rev. Sci. Instrum. 2004, 75, 4481-4486.

9. Gaal, M.; Gadermaier, C.; Plank, H.; Moderegger, E.; Pogantsch, A.; Leising, G.; List, E.J. Imprinted conjugated polymer laser. Adv. Mater. 2003, 15, 1165-1167.

10. Wenger, B.; Tétreault, N.; Welland, M.E.; Friend, R.H. Mechanically tunable conjugated polymer distributed feedback lasers. Appl. Phys. Lett. 2010, 97, 237.

11. Namdas, E.B.; Tong, M.; Ledochowitsch, P.; Mednick, S.R.; Yuen, J.D.; Moses, D.; Heeger, A.J. Low thresholds in polymer lasers on conductive substrates by distributed feedback nanoimprinting: Progress toward electrically pumped plastic lasers. Adv. Mater. 2009, 21, 799-802.

12. Klinkhammer, S.; Woggon, T.; Geyer, U.; Vannahme, C.; Dehm, S.; Mappes, T.; Lemmer, U. A continuously tunable low-threshold organic semiconductor distributed feedback laser fabricated by rotating shadow mask evaporation. Appl. Phys. B 2009, 97, 787.

13. Tsiminis, G.; Wang, Y.; Kanibolotsky, A.L.; Inigo, A.R.; Skabara, P.J.; Samuel, I.D.; Turnbull, G.A. Nanoimprinted organic semiconductor laser pumped by a light-emitting diode. Adv. Mater. 2013, 25, 2826-2830.

14. Schauer, S.; Liu, X.; Worgull, M.; Lemmer, U.; Hölscher, H. Shape-memory polymers as flexible resonator substrates for continuously tunable organic DFB lasers. Opt. Mater. Express 2015, 5, 576-584.

15. Ge, C.; Lu, M.; Jian, X.; Tan, Y.; Cunningham, B.T. Large-area organic distributed feedback laser fabricated by nanoreplica molding and horizontal dipping. Opt. Express 2010, 18, 12980-12991.

16. Herrnsdorf, J.; Guilhabert, B.; Chen, Y.; Kanibolotsky, A.L.; Mackintosh, A.; Pethrick, R.; Skabara, P.; Gu, E.; Laurand, N.; Dawson, M. Flexible blue-emitting encapsulated organic semiconductor DFB laser. Opt. Express 2010, 18, 25535-25545.

17. Xia, R.; Heliotis, G.; Stavrinou, P.; Bradley, D. Polyfluorene distributed feedback lasers operating in the green-yellow spectral region. Appl. Phys. Lett. 2005, 87, 031104.

18. Heliotis, G.; Xia, R.; Turnbull, G.A.; Andrew, P.; Barnes, W.L.; Samuel, I.D.W.; Bradley, D.D. Emission characteristics and performance comparison of polyfluorene lasers with one-and two-dimensional distributed feedback. Adv. Funct. Mater. 2004, 14, 91-97. 
19. Camposeo, A.; Del Carro, P.; Persano, L.; Pisignano, D. Electrically tunable organic distributed feedback lasers embedding nonlinear optical molecules. Adv. Mater. 2012, 24, OP221-OP225.

20. Voss, T.; Scheel, D.; Schade, W. A microchip-laser-pumped DFB-polymer-dye laser. Appl. Phys. B 2001, 73, 105-109.

21. Boj, P.G.; Morales-Vidal, M.; Villalvilla, J.M.; Quintana, J.A.; Marcilla, A.; Díaz-García, M.A. Organic distributed feedback laser to monitor solvent extraction upon thermal annealing in solution-processed polymer films. Sens. Actuators B Chem. 2016, $232,605-610$.

22. Quintana, J.A.; Villalvilla, J.M.; Morales-Vidal, M.; Boj, P.G.; Zhu, X.; Ruangsupapichat, N.; Tsuji, H.; Nakamura, E.; Díaz-García, M.A. An Efficient and Color-Tunable Solution-Processed Organic Thin-Film Laser with a Polymeric Top-Layer Resonator. Adv. Opt. Mater. 2017, 5, 1700238.

23. Görrn, P.; Lehnhardt, M.; Kowalsky, W.; Riedl, T.; Wagner, S. Elastically Tunable Self-Organized Organic Lasers. Adv. Mater. 2011, 23, 869-872.

24. Döring, S.; Kollosche, M.; Rabe, T.; Stumpe, J.; Kofod, G. Electrically tunable polymer DFB laser. Adv. Mater. 2011, 23, 4265-4269.

25. Klinkhammer, S.; Liu, X.; Huska, K.; Shen, Y.; Vanderheiden, S.; Valouch, S.; Vannahme, C.; Bräse, S.; Mappes, T.; Lemmer, $\mathrm{U}$. Continuously tunable solution-processed organic semiconductor DFB lasers pumped by laser diode. Opt. Express $\mathbf{2 0 1 2}$ 20, 6357-6364.

26. Wang, J.; Weimann, T.; Hinze, P.; Ade, G.; Schneider, D.; Rabe, T.; Riedl, T.; Kowalsky, W. A continuously tunable organic DFB laser. Microelectr. Eng. 2005, 78, 364-368.

27. Čehovski, M.; Becker, J.; Charfi, O.; Johannes, H.H.; Müller, C.; Kowalsky, W. Single-Mode Polymer Ridge Waveguide Integration of Organic Thin-Film Laser. Appl. Sci. 2020, 10, 2805.

28. Ozaki, R.; Shinpo, T.; Yoshino, K.; Ozaki, M.; Moritake, H. Tunable liquid crystal laser using distributed feedback cavity fabricated by nanoimprint lithography. Appl. Phys. Express 2008, 1, 012003.

29. Klinkhammer, S.; Heussner, N.; Huska, K.; Bocksrocker, T.; Geislhöringer, F.; Vannahme, C.; Mappes, T.; Lemmer, U. Voltagecontrolled tuning of an organic semiconductor distributed feedback laser using liquid crystals. Appl. Phys. Lett. 2011, 99, 137.

30. Schäfer, F.P.; Schmidt, W.; Volze, J. Organic dye solution laser. Appl. Phys. Lett. 1966, 9, 306-309.

31. Zhai, T.; Zhang, X.; Pang, Z. Polymer laser based on active waveguide grating structures. Opt. Express 2011, 19, 6487-6492.

32. McGehee, M.D.; Gupta, R.; Veenstra, S.; Miller, E.K.; Diaz-Garcia, M.A.; Heeger, A.J. Amplified spontaneous emission from photopumped films of a conjugated polymer. Phys. Rev. B 1998, 58, 7035.

33. Van den Berg, S.; den Bezemer, R.v.S.; Schoo, H.; Eliel, E. From amplified spontaneous emission to laser oscillation: Dynamics in a short-cavity polymer laser. Opt. Lett. 1999, 24, 1847-1849.

34. Bonal, V.; Muñoz-Mármol, R.; Gámez, F.G.; Morales-Vidal, M.; Villalvilla, J.M.; Boj, P.G.; Quintana, J.A.; Gu, Y.; Wu, J.; Casado, J. Solution-processed nanographene distributed feedback lasers. Nat. Commun. 2019, 10, 3327.

35. Bonal, V.; Quintana, J.A.; Villalvilla, J.M.; Boj, P.G.; Díaz-García, M.A. Controlling the emission properties of solution-processed organic distributed feedback lasers through resonator design. Sci. Rep. 2019, 9, 11159.

36. Simović, A.; Drljača, B.; Savović, S.; Djordjevich, A.; Min, R. Investigation of bandwidth in multimode graded-index plastic optical fibers. Opt. Express 2021, 29, 29587-29594.

37. Peters, K. Polymer optical fiber sensors-A review. Smart Mater. Struct. 2010, 20, 013002.

38. Savović, S.; Drljača, B.; Kovačević, M.S.; Djordjevich, A.; Bajić, J.S.; Stupar, D.Z.; Stepniak, G. Frequency response and bandwidth in low-numerical-aperture step-index plastic optical fibers. Appl. Opt. 2014, 53, 6999-7003.

39. He, J.; Chan, W.K.; Cheng, X.; Tse, M.L.; Lu, C.; Wai, P.K.; Savovic, S.; Tam, H.Y. Experimental and theoretical investigation of the polymer optical fiber random laser with resonant feedback. Adv. Opt. Mater. 2018, 6, 1701187.

40. Kuriki, K.; Kobayashi, T.; Imai, N.; Tamura, T.; Koike, Y.; Okamoto, Y. Organic dye-doped polymer optical fiber laser. Polym. Adv. Technol. 2000, 11, 612-616.

41. Yuyama, S.; Nakajima, T.; Yamashita, K.; Oe, K. Solid state organic laser emission at $970 \mathrm{~nm}$ from dye-doped fluorinated-polyimide planar waveguides. Appl. Phys. Lett. 2008, 93, 252.

42. Mhibik, O.; Leang, T.; Siove, A.; Forget, S.; Chénais, S. Broadly tunable $(440-670 \mathrm{~nm})$ solid-state organic laser with disposable capsules. Appl. Phys. Lett. 2013, 102, 041112.

43. Arrue, J.; Jiménez, F.; Ayesta, I.; Illarramendi, M.A.; Zubia, J. Polymer-optical-fiber lasers and amplifiers doped with organic dyes. Polymers 2011, 3, 1162-1180.

44. Spelthann, S.; Unland, S.; Thiem, J.; Jakobs, F.; Kielhorn, J.; Ang, P.Y.; Johannes, H.H.; Kracht, D.; Neumann, J.; Ruehl, A. Towards Highly Efficient Polymer Fiber Laser Sources for Integrated Photonic Sensors. Sensors 2020, 20, 4086.

45. Kuriki, K.; Kobayashi, T.; Imai, N.; Tamura, T.; Nishihara, S.; Nishizawa, Y.; Tagaya, A.; Koike, Y.; Okamoto, Y. High-efficiency organic dye-doped polymer optical fiber lasers. Appl. Phys. Lett. 2000, 77, 331-333.

46. Samuel, I.D.; Namdas, E.B.; Turnbull, G.A. How to recognize lasing. Nat. Photon. 2009, 3, 546-549.

47. Lemmer, U. Stimulated emission and lasing in conjugated polymers. Polym. Adv. Technol. 1998, 9, $476-487$. 
48. Miniscalco, W.J.; Thompson, B.A.; Dakss, M.L.; Zemon, S.A.; Andrews, L.J. Measurement and analysis of cross sections for rare-earth-doped glasses. In Fiber Laser Sources and Amplifiers III; International Society for Optics and Photonics: Bellingham, WA, USA, 1992; Volume 1581, pp. 80-90.

49. Malinin, A.; Zanishevskaja, A.; Tuchin, V.; Skibina, Y.S.; Silokhin, I.Y. Photonic crystal fibers for food quality analysis. In Photonic Solutions for Better Health Care III; International Society for Optics and Photonics: Bellingham, WA, USA, 2012; Volume 8427, p. 842746 